\title{
Incontinence pessaries: size, POPQ measures, and successful fitting
}

\author{
Charles W. Nager • Holly E. Richter • Ingrid Nygaard • \\ Marie Fidela Paraiso • Jennifer M. Wu • \\ Kimberly Kenton • Shanna D. Atnip • Cathie Spino • \\ for the Pelvic Floor Disorders Network (PFDN)
}

Received: 14 November 2008 / Accepted: 11 March 2009/Published online: 16 June 2009

(C) The Author(s) 2009. This article is published with open access at Springerlink.com

\begin{abstract}
Introduction and hypothesis The aim of the study was to determine whether successful incontinence pessary fitting or pessary size can be predicted by specific POPQ measurements in women without advanced pelvic organ prolapse.

Methods In a multicenter study, women with stress urinary incontinence (SUI) and POPQ stage $\leq 2$ were randomized to three treatment arms: (1) incontinence pessary, (2) behavioral therapy, or (3) both. This study evaluates incontinence pessary size, POPQ measures, and successful fitting in the 266 women assigned to treatment arms 1 and 3.
\end{abstract}

Supported by grants from the Eunice Kennedy Shriver National Institute of Child Health and Human Development and the NIH Office of Research on Women's Health at National Institutes of Health (U10 HD54215, U10 HD41267, U10 HD41250, U10 HD41261, U10 HD54214, U10 HD54241, U10 HD54136, and U01 HD41249).

\section{W. Nager}

Department of Reproductive Medicine, University of California,

San Diego,

San Diego, CA, USA

\section{H. E. Richter}

Department of Obstetrics and Gynecology,

University of Alabama at Birmingham,

Birmingham, AL, USA

\section{Nygaard}

Department of Obstetrics and Gynecology, University of Utah,

Salt Lake City, UT, USA

M. Fidela Paraiso

Obstetrics, Gynecology, and Women's Health Institute,

Cleveland Clinic,

Cleveland, $\mathrm{OH}$, USA

J. M. Wu

Department of Obstetrics and Gynecology,

Duke University Medical Center,

Durham, NC, USA
Results Two hundred thirty-five women (92\%) were successfully fitted with an incontinence ring $(n=122)$ or dish $(n=113)$. Hysterectomy, genital hiatus $(\mathrm{GH})$, and $\mathrm{GH} /$ total vaginal length (TVL) ratios did not predict unsuccessful fitting $(p>0.05)$. However, mean TVL was greater in women successfully fitted (9.6 vs. $8.8 \mathrm{~cm}, p<0.01)$. Final pessary diameter was not predicted by TVL, point $\mathrm{D}$, or point $\mathrm{C}(p>0.05)$.

Conclusions The vast majority of women with SUI can be successfully fitted with an incontinence pessary, but specific POPQ measures were not helpful in determining incontinence pessary size.

\section{K. Kenton}

Department of Obstetrics and Gynecology,

Loyola University,

Maywood, IL, USA

S. D. Atnip

Department of Obstetrics and Gynecology,

University of Texas, Southwestern,

Dallas, TX, USA

C. Spino

Department of Biostatistics, University of Michigan,

Ann Arbor, MI, USA

C. W. Nager $(\square)$

UCSD Women's Pelvic Medicine Center,

9350 Campus Point Drive, Perlman Suite 2A, MC 8433,

La Jolla, CA 92037-8433, USA

e-mail: cnager@ucsd.edu 
Keywords Incontinence ring · Incontinence dish · Pessary · Stress urinary incontinence

$\begin{array}{ll}\text { Abbreviations } \\ \text { POPQ } & \text { Pelvic organ prolapse quantification } \\ \text { SUI } & \text { Stress urinary incontinence } \\ \text { GH } & \text { Genital hiatus } \\ \text { TVL } & \text { Total vaginal length } \\ \text { ATLAS } & \begin{array}{l}\text { Ambulatory Treatments for Leakage Associated } \\ \end{array} \\ & \text { with Stress }\end{array}$

\section{Introduction}

The vaginal pessary has been used for centuries as a conservative treatment of pelvic organ prolapse [1] and more recently for stress urinary incontinence [2-4]. Pessary fitting has long been considered an art rather than a science, a process of trial and error whereby the clinician's training and experience best predict success. The subjective nature of pessary fitting comes from little or no formal clinical training. Furthermore, there is no consensus on clinical indications for various types of pessaries, patient characteristics for sizing, or appropriate pessary care [5,6]. Manufacturer product information [7] and texts give general guidelines for pessary fitting; however, these recommendations are not supported by research.

No studies to date have consistently linked specific patient characteristics to pessary size. A scientific prediction of successful incontinence pessary fitting based on a quantifiable prolapse exam could remove our biases of who will or will not be successful, decrease patient and clinician time needed for fitting, and serve as a useful tool in counseling patients regarding treatment options. Predicting size may give clinicians the ability to maintain a smaller selection of pessary sizes or types in the office, which could reduce office costs.

The primary aim of this study was to determine, in a group of women without advanced pelvic organ prolapse and with or without a uterus, (1) whether successful incontinence pessary fitting can be predicted by specific pelvic organ prolapse quantification (POPQ) measurements [8] and (2) whether POPQ measures predict pessary size. We hypothesized that total vaginal length (TVL) correlates with pessary diameter during successful pessary fitting. We also hypothesized that previous hysterectomy (by any route) and a large genital hiatus impair the ability to retain a pessary.

\section{Materials and methods}

This analysis was based on a subset of subjects enrolled in the Ambulatory Treatments for Leaking Associated with
Stress (ATLAS) trial, a randomized multicenter study of the Pelvic Floor Disorders Network. Women with stress urinary incontinence (SUI) and POPQ stage $\leq 2$ were randomized to one of three treatment arms: (1) incontinence pessary; (2) behavioral therapy, including pelvic floor muscle training and exercise and bladder control strategies; or (3) a combination of the two treatments. IRB approval was obtained for all participating sites, and all eligible subjects provided informed consent before beginning the study. Full details on the study design and methodology have been previously published [9].

Seven hundred forty-one subjects enrolled in ATLAS during the period May 2005 to November 2007. Four hundred forty-five subjects were randomized. This analysis includes the 266 women assigned to receive an incontinence pessary (treatment arms 1 and 3). At the time of study enrollment, a POPQ exam was performed as described by Bump et al. [8] by a physician or study nurse who regularly performs these exams. Genital hiatus $(\mathrm{GH})$ was measured at rest and with Valsalva during the POPQ exam; as the measures were not markedly different, for the purpose of this study, we used the GH with Valsalva measures.

Pessary fittings were performed by an interventionist in the study who also performed the behavioral therapy in the study. This interventionist was a trained nurse practitioner, nurse, or physical therapist who did not perform the POPQ assessment and was not aware of the POPQ result. The type of incontinence pessary (ring or dish-see Fig. 1) used for each subject was left to the discretion of the interventionist. The size and type of the final fitted pessary were recorded at all visits. Proper size and adequate fitting was considered when the provider could place a finger between the pessary and the vaginal walls and after fitting the subject could stand, cough, and strain with the pessary retained. Multiple pessaries could be fitted at one visit to determine the correct size for subject use. A pessary fitting was considered unsuccessful if the interventionist failed to obtain an adequate fit after at least three attempts, the participant found the pessary painful, or the participant did not plan to use the pessary after fitting.

For calculations of incontinence pessary diameter, we used the recorded size and type of the final fitted pessary from the last fitting visit. We obtained the corresponding outer diameter of this ring or dish from data provided by the Milex website at http://www.milexproducts.com. Incontinence rings are typically sized in small integers ranging from size no. 0 (1.75 in./44 $\mathrm{mm}$ diameter) to size no. 10 (4.25 in./108 $\mathrm{mm}$ diameter) with 0.25 in. $(6-7 \mathrm{~mm})$ diameter differences between integer sizes. Incontinence dishes are not measured as integer sizes, but instead are described by their diameter; they start at a 55-mm-diameter size and increase in $5 \mathrm{~mm}$ intervals larger, up to a maximum size of $85 \mathrm{~mm}$ diameter. Seven millimeters was added to each corresponding ring diameter to account for 


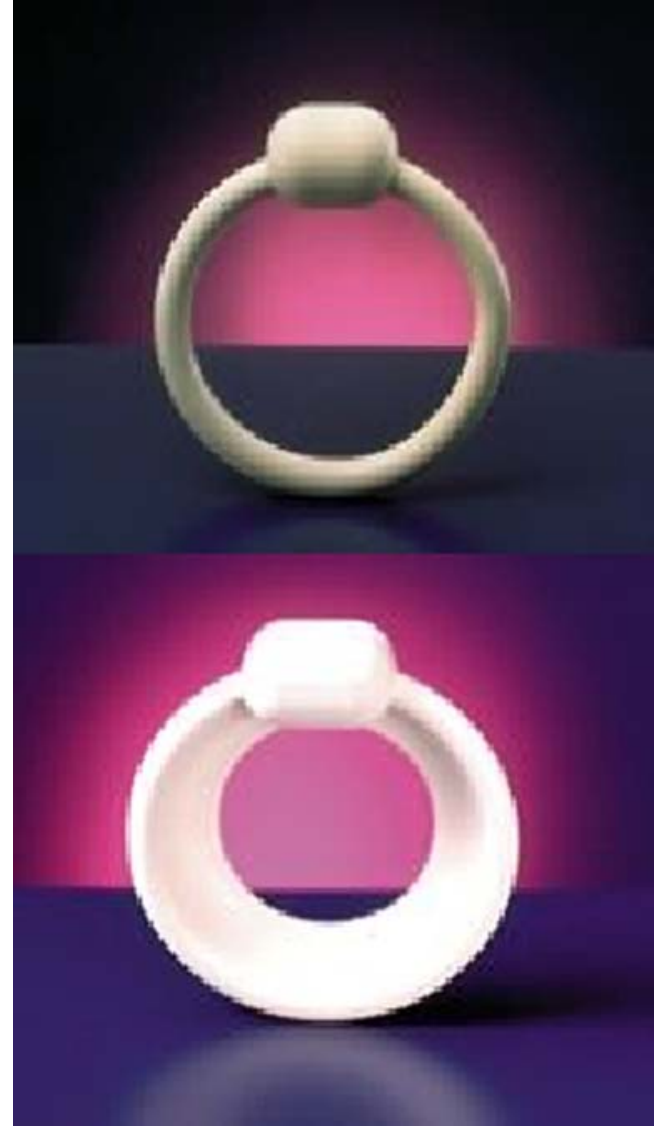

Fig. 1 Incontinence ring and incontinence dish (courtesy of Milex web site)

the increased outside diameter produced by the knob on the incontinence ring or dish.

Comparisons of characteristics between subjects with successful and unsuccessful pessary fittings were tested using two-sample $t$ tests for continuous outcomes and Fisher's exact tests for discrete outcomes. Linear regression was used to explore the relationship between POPQ measures and final pessary diameter in women with and without a cervix (hysterectomy group). Both linear and quadratic models were investigated. All analyses were performed using SAS 9.1 (Cary, NC, USA).

\section{Results}

The study sample includes 266 women in the ATLAS study who were randomized to one of the pessary treatment arms. Data on key variables were complete, ranging from $>99 \%$ ( 265 of 266 for hysterectomy status) to $92 \%$ ( 245 of 266 for final pessary size). The descriptive characteristics of the study population are found in Table 1 . The mean age of women in this study was 49 years (range, 19 to 81 years), with a mean body mass index of $29 \mathrm{~kg} / \mathrm{m}^{2}$ (range, 18.0 to 58.6). The majority of study participants were parous
(94\%). Fifty-nine (22\%) had a prior total hysterectomy (no cervix and therefore no point D on POPQ exam). Relatively few women had surgeries for urinary incontinence $(5 \%)$ or prolapse $(3 \%)$.

Forty-one percent (104) of the women had stage 2, 49\% had stage 1 , and $10 \%$ had stage 0 POPQ prolapse. The group POPQ measures were (mean $\pm \mathrm{SD}$ in $\mathrm{cm}$ ): TVL (9.5 \pm 1.1); C (-7.2 \pm 2.1$)$; D (-7.1 \pm 4.9$)$; and GH (3.1 \pm 1.0$)$. The $\mathrm{GH} / \mathrm{TVL}$ ratio was $0.33 \pm 0.11$ (mean $\pm \mathrm{SD}$ ).

During the first visit, 256 subjects attempted a pessary fitting. Fifty-four percent were fit during the first visit, $36 \%$ needed a second pessary fitting visit, and $10 \%$ required a third and final visit. During the first visit, 104 women (41\%) required only one pessary for fitting, while the remaining women were fit with more than one pessary (range, two to nine different pessaries). The subjects had a median of two pessaries attempted over the course of the

Table 1 Descriptive statistics of the 266 subjects assigned to receive an incontinence pessary

\begin{tabular}{lc}
\hline Characteristic & $N(\%)$ or mean (SD), range \\
\hline Subject characteristics & $49(11), 19$ to 81 \\
Age & $29.5(6.8), 18.0$ to 58.6 \\
BMI $\left(\mathrm{kg} / \mathrm{m}^{2}\right)$ & $75(28 \%)$ \\
BMI normal $\left(<25 \mathrm{~kg} / \mathrm{m}^{2}\right)$ & $83(31 \%)$ \\
BMI overweight $\left(25-29.9 \mathrm{~kg} / \mathrm{m}^{2}\right)$ & $107(40 \%)$ \\
BMI obese $\left(\geq 30 \mathrm{~kg} / \mathrm{m}^{2}\right)$ & $250(94 \%)$ \\
Parous & $59(22 \%)$ \\
Prior hysterectomy & $13(5 \%)$ \\
Prior surgical treatment for pelvic & $7(3 \%)$ \\
organ prolapse & \\
Prior surgical treatment for urinary & \\
incontinence & $26(10 \%)$ \\
POPQ parameters & $127(49 \%)$ \\
Stage 0 & $104(41 \%)$ \\
Stage 1 & $9.5(1.1), 6$ to 12 \\
Stage 2 & $-7.2(2.1),-12$ to -5 \\
TVL (cm) & $-7.1(4.9),-12$ to -1 \\
C (cm) & $3.1(1.0), 1$ to 7 \\
D (cm) & $0.33(0.11), 0.09$ to 0.88 \\
GH (cm) & \\
GH/TVL ratio & \\
Pessary variables ${ }^{\mathrm{a}}$ & \\
Final type of pessary & \\
Incontinence ring with support & \\
Incontinence dish & \\
Final pessary size $($ diameter, mm) & \\
Plan to use pessary (final status) & $(8 \%)$ \\
Yes & \\
No & \\
\hline
\end{tabular}

${ }^{\text {a }}$ Pessary variables are provided for the final fitted pessary (with a maximum of three possible visits to fit the pessary) 
one to three visits. Approximately half of the women had an incontinence ring with support as their final pessary $(52 \%)$, while the remainder had an incontinence dish (48\%). In women with stage 2 prolapse, the incontinence dish was used $53 \%$ of the time and the ring $47 \%$ of the time, but this difference was not significant. In women with incontinence rings as their final pessary, the average diameter of the final fitted pessary was $69 \mathrm{~mm}$ ( $\mathrm{SD}=$ $8 \mathrm{~mm}$ ), which is approximately a size 3, while in women with dishes as their final fitted pessary, the average diameter was $74 \mathrm{~mm}$. ( $\mathrm{SD}=8 ; p<0.01)$.

Two hundred thirty-five women (92\%) were successfully fitted with an incontinence ring $(n=122)$ or incontinence dish ( $n=113) ; 20$ women $(8 \%)$ were unsuccessfully fitted. The reasons for unsuccessful fitting (not mutually exclusive) were categorized as follows: unwilling to use (nine), not fitting (seven), pain (seven), and other (three). Table 2 displays the relevant POPQ variables or hysterectomy status in women with successful and unsuccessful final pessary fitting. Mean TVL was greater in women successfully fitted $(9.6 \mathrm{~cm})$ compared to those unsuccessfully fitted ( $8.8 \mathrm{~cm} ; p<0.01)$. Hysterectomy, GH, and GH/TVL ratios did not predict unsuccessful fitting $(p>0.05)$. Graphs of pessary diameter and TVL for women with a cervix (Fig. 2) and for women after total hysterectomy (Fig. 3) demonstrate no obvious relationship between these two variables. TVL, point $\mathrm{D}$, and point $\mathrm{C}$ for women with and without a prior hysterectomy were not predictive of final pessary diameter in women with successful pessary fittings $(p>0.05$; Table 3$)$ Similar negative results were achieved using linear or quadratic models with all study subjects (i.e., women with successful or unsuccessful pessary fittings; data not shown).

\section{Discussion}

We found that the vast majority of women $(92 \%)$ with SUI without advanced pelvic organ prolapse can be successfully fitted with an incontinence pessary by trained nurse practitioners, RNs, or physical therapists. Previous hyster-

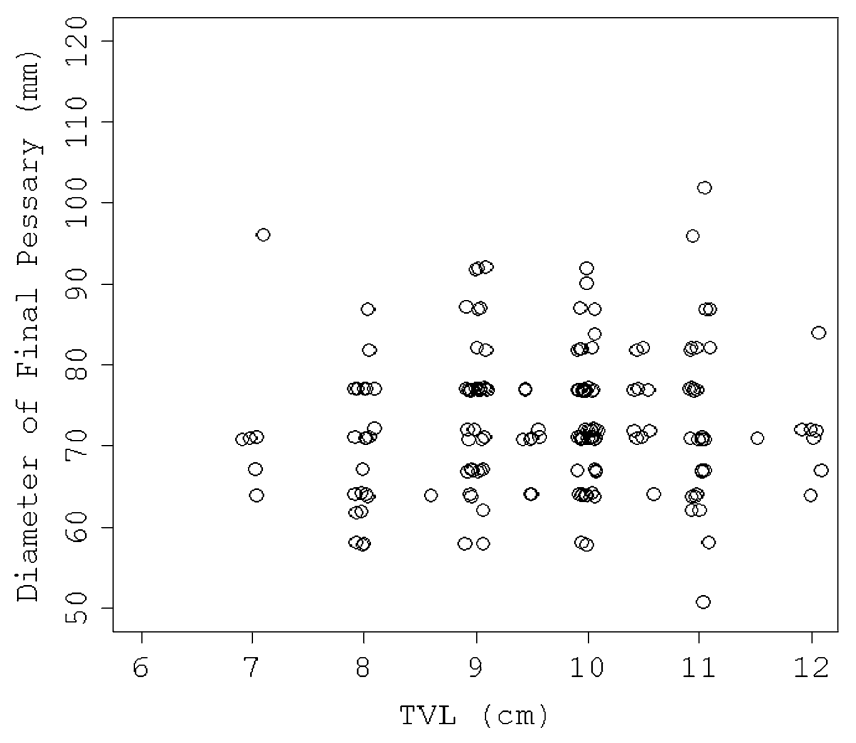

Fig. 2 Pessary diameter and TVL for women with cervix intact $(n=$ 173)

ectomy did not result in a higher rate of unsuccessful incontinence pessary fit. Women with a longer total vaginal length were more likely to be successfully fitted but various formulas involving total vaginal length did not predict pessary size. In fact, no vaginal measurement per POPQ evaluation proved helpful in determining pessary size. Approximately two thirds of our subjects could be fitted with a no. 2 , no. 3 , or no. 4 incontinence ring or a 65-, 70-, or $75-\mathrm{mm}$ incontinence dish. This information may be useful to the clinician for office inventory supply.

A few studies have described patient characteristics associated with continued pessary use in prolapse or prolapse and incontinent patients. Clemons et al. [10] found that advanced age was associated with pessary continuation, but that stage III-IV prolapse and desire for surgery were associated with discontinuation. Wu et al. [11] found that patients with both pelvic organ prolapse and stress incontinence were more likely to chose surgery, but that advanced prolapse was not associated with pessary discontinuation.
Table 2 POPQ variables or hysterectomy status in women with successful and unsuccessful final pessary fitting

\footnotetext{
${ }^{\text {a }}$ Derived from two-sample $t$ test for continuous outcomes and from Fisher's exact test for discrete outcomes
}

\begin{tabular}{lcccccr}
\hline Variable & \multicolumn{2}{l}{ Successful final pessary fitting } & & \multicolumn{2}{l}{ Unsuccessful final pessary fitting } & \multirow{2}{*}{$p$ value $^{\mathrm{a}}$} \\
\cline { 2 - 3 } & $N$ & Mean (SE) or $n(\%)$ & & $N$ & Mean (SE) or $n(\%)$ & \\
\hline TVL & 229 & $9.6(0.07)$ & & 20 & $8.8(0.24)$ & $<0.01$ \\
GH & 233 & $3.1(0.06)$ & & 20 & $3.2(0.33)$ & 0.68 \\
GH/TVL ratio & 227 & $0.33(0.01)$ & & 20 & $0.38(0.04)$ & 0.16 \\
Ba & 234 & $-1.65(0.06)$ & & 20 & $-1.60(0.26)$ & 0.82 \\
Bp & 234 & $-2.25(0.05)$ & & 20 & $-2.00(0.22)$ & 0.22 \\
C & 235 & $-7.15(0.14)$ & & 20 & $-7.00(0.36)$ & 0.75 \\
Hysterectomy & 234 & $54(23 \%)$ & & 20 & $4(20 \%)$ & 0.38 \\
\hline
\end{tabular}




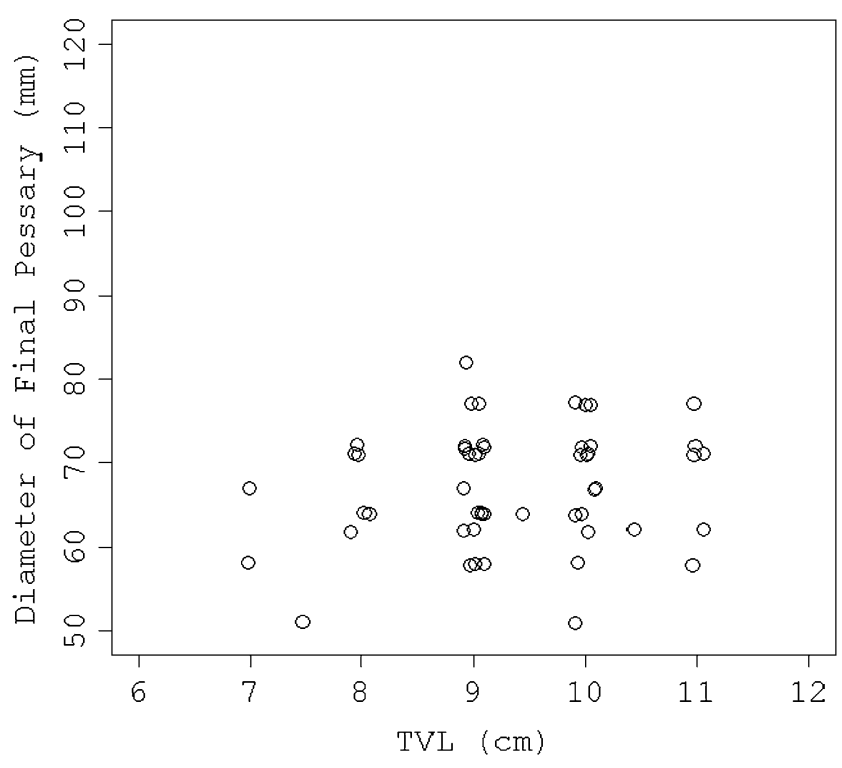

Fig. 3 Pessary diameter and TVL for women with total hysterectomy $(n=54)$

There are limited data regarding specific vaginal dimensions that may be associated with successful pessary fitting. A widened genital introitus, short vaginal length, and posterior compartment defects [10,12-14] have been associated with unsuccessful fitting in women with pelvic organ prolapse and incontinence. In one study, it was hypothesized that women with a wider genital hiatus would be more difficult to fit successfully as incontinence pessaries are somewhat dependent on the presence of levator support to stay in place [15]. However, when utilizing POPQ measures of the $\mathrm{GH}$, there was no difference in the proportion of women that were successfully fit whether the GH measured $<3 \mathrm{~cm}$ or $>3 \mathrm{~cm}(p=1.0)$.

There is no uniform definition for successful pessary fitting. We considered fitting to be unsuccessful if the provider could not obtain an adequate fit after at least three attempts, if the woman found the pessary painful, or if she did not plan to use the pessary after fitting. Others have considered fitting successful when the pessary was comfortable and retained during Valsalva and voiding at the initial visit [13] or when the fit was appropriate and the woman continued to use the pessary at the 3-week follow- up visit [16]. Whatever definition is utilized, in order to optimize pessary use in women that desire this means of treatment, early and consistent long-tem follow-up should be performed $[17,18]$.

While previous hysterectomy was identified in one study as a risk factor for unsuccessful fitting in women with prolapse [13], we did not find this to be the case in our population of women without advanced prolapse. Women using pessaries to treat stress incontinence differ from women using them to treat pelvic organ prolapse. The population of women with stress incontinence is younger, is less likely to have had previous surgeries, and has a longer vaginal length and narrower genital hiatus than the population with prolapse. Accordingly, the success rate for fitting pessaries in women with SUI $(92 \%$ in our study, $89 \%$ in another population of women with SUI [15]) is generally higher than that for women with advanced prolapse (41\% [16], 73\% [10], and 90\% [19]).

A strength of this study was that as part of a randomized non-surgical treatment trial, all patients underwent a standardized baseline evaluation, including the POPQ evaluation. An exhaustive effort was made to successfully fit and follow-up with these patients; therefore, there was minimal loss to follow-up early in the process, which is important in carefully characterizing those subjects that were fitting failures. The major limitation of this study is that the results and conclusions of our study are limited to women with SUI and no significant prolapse; a group of women with more prolapse or a wider range of POPQ values may have different results. Another limitation of this study was the lack of utilization of other patient characteristics in order to characterize those that were fitting successes or failures. The primary purpose of this study was to determine whether successful pessary fitting could be predicted by specific POPQ measures and whether these measures could also predict pessary size. As this was not the case, clearly the performance of a POPQ examination prior to pessary fitting in women with stress predominant urinary incontinence without advanced prolapse is not necessary. The vaginal measures of the POPQ exam include the sagittal axis of the vagina, but not the lateral or coronal dimensions of the vagina, and these later measures may be more important for fitting.
Table 3 Relationship between POPQ parameters and pessary diameter Linear regression
pessary diameter

\begin{tabular}{llccc}
\hline POPQ parameter & Sample & Samples & Regression coefficient (SE) & $p$ value \\
\hline TVL & Hysterectomy & 54 & $1.32(0.95)$ & 0.17 \\
& No hysterectomy & 173 & $0.49(0.57)$ & 0.39 \\
D & No hysterectomy & 177 & $-0.04(0.41)$ & 0.93 \\
C & Hysterectomy & 54 & $-0.89(0.71)$ & 0.21 \\
& No hysterectomy & 177 & $-0.25(0.29)$ & 0.38 \\
\hline
\end{tabular}


We conclude that incontinence pessary fitting remains an art rather than a science. Fortunately, however, it is an art that is easy to do: Most women were successfully fitted, and most did not need to try more than two pessary sizes to achieve the appropriate fit.

Given the lack of correlation with anatomic and baseline characteristics, these data suggest that incontinence pessary fitting can occur in a primary care setting by interested and experienced providers of all levels, including nurses, nurse practitioners, physical therapists, and physicians.

\section{Conflicts of interest None.}

Open Access This article is distributed under the terms of the Creative Commons Attribution Noncommercial License which permits any noncommercial use, distribution, and reproduction in any medium, provided the original author(s) and source are credited.

\section{References}

1. Shah SM, Sultan AH, Thakar R (2006) The history and evolution of pessaries for pelvic organ prolapse. Int Urogynecol J 17:170175

2. Nygaard I (1995) Prevention of exercise incontinence with mechanical devices. J Reprod Med 40:89-94

3. Suarez GM, Baum NH, Jacobs J (1991) Use of standard contraceptive diaphragm in management of stress urinary incontinence. Urology 37:119-122

4. Realini JP, Walters MD (1990) Vaginal diaphragm rings in the treatment of stress urinary incontinence. J Am Board Fam Pract 3:99-103

5. Pott-Grinstein E, Newcomer JR (2001) Gynecologists' patterns of prescribing pessaries. J Reprod Med 46:205-208

6. Cundiff GW, Weidner AC, Visco AG, Bump RC, Addison W (2000) A survey of pessary use by members of the American Urogynecological Society. Obstet Gynecol 95:931-935
7. Cooper Surgical Product Catalog. Chapter on Milex Silicone Pessaries. 80973 Catalog QZ, Rev 1/06. Published by Cooper Surgical, Trumbull, CT. USA

8. Bump RC, Mattiasson A, Bo K, Brubaker L, DeLancey JO, Klarskov P, Shull BL, Smith AR (1996) The standardization of terminology of female pelvic organ prolapse and pelvic floor dysfunction. Am J Obstet Gynecol 175:10-17

9. Richter HE, Burgio KL, Goode PS, Borello-France D, Bradley CS, Brubaker L, Handa V, Kieback D, Visco AG, Zyczynski HM, Wei JT, Weber AM, for the Pelvic Floor Disorders Network (2007) Non-surgical management of stress urinary incontinence: ambulatory treatments for leakage associated with stress incontience (ATLAS) trial. Clin Trials 4:92-101

10. Clemons JL, Aguilar VC, Tillinghast TA, Jackson ND, Myers DL (2004) Risk factors associated with an unsuccessful pessary fitting trial in women with pelvic organ prolapse. Am J Obstet Gynecol 190:345-350

11. Wu V, Farrell SA, Baskett TF, Flowerdew G (1997) A simplified protocol for pessary management. Obstet Gynecol 90:990-994

12. Nguyen JN, Jones CR (2005) Pessary treatment of pelvic relaxation. J Wound Ostomy Continence Nurs 32:255-261

13. Maito JM, Quam ZA, Craig E, Danner KA, Rogers RG (2006) Predictors of successful pessary fitting and a continued use in a nurse-midwifery pessary clinic. J Midwifery Wom Health 51:78-84

14. Hanson LA, Schulz JA, Flood CG, Cooley B, Tam F (2006) Vaginal pessaries in managing women with pelvic organ prolapse and urinary incontinence: patient characteristics and factors contributing to success. Int Urogynecol J Pelvic Floor Dysfunct 17:155-159

15. Donnelly MJP-MS, Olsen AL, Nygaard IE (2004) Vaginal pessaries for the management of stress and mixed urinary incontinence. Int Urogynecol J Pelvic Floor Dysfunct 15:302-307

16. Mutone MF, Terry C, Hale DS, Benson JT (2005) Factors which influence the short-term success of pessary management of pelvic organ prolapse. Am J Obstet Gynecol 193:89-94

17. Farrell SA, Singh B, Aldakhil L (2004) Continence pessaries in the management of urinary incontinence in women. J Obstet Gynaecol Can 26:113-117

18. Palumbo MV (2000) Pessary placement and management. Ostomy Wound Manage 46:40-45

19. Cundiff GW AC, Bent AE, Coates KW, Schaffer JI, Strohbehn K, Handa VL (2007) The PESSRI Study: symptom relief outcomes of a randomized crossover trial of the ring and Gellhourn pessaries. Am J Obstet Gynecol 196:405.e401-405.e408 